death recorded in their GP record. Where cause of death was obtained from national records $(n=163)$, primary causes were MI $(n=29,17.8 \%)$ and pneumonia $(n=23,14 \%)$. Mean age at death was $77.7 \pm 9.3$ years. Mortality was significantly higher among patients who were older at baseline. Gender, diabetes type, smoking status and clinical parameters at baseline were not significant predictors of mortality.

Discussion Improvements in the clinical profile of patients enrolled in the programme since its introduction suggests primarycare-led integrated diabetes management can perform favourably in the long-term. However, the high incidence of macrovascular complications, prevalence of retinopathy and mortality rate indicates the importance of effective management.

\section{OP16 CHALLENGES EXPERIENCED BY COMMUNITY-BASED CLINICAL NURSE SPECIALISTS IN SUPPORTING THE DELIVERY OF INTEGRATED DIABETES CARE: A QUALITATIVE STUDY}

F Riordan*, SM McHugh, PM Kearney. Department of Epidemiology and Public Health, University College Cork, Cork, Ireland

\subsection{6/jech-2017-SSMAbstracts. 16}

Background In Ireland, more Diabetes Nurse Specialists (DNSs) have been introduced into the community as part of a national programme to standardise and improve diabetes care. DNSs support the delivery of a new model of care whereby uncomplicated type 2 diabetes (T2DM) is managed in primary care, and complicated T2DM is managed between primary and secondary care. Historically diabetes care in Ireland has often been delivered in an unstructured way, lacking integration between primary and secondary care. Given this context we wanted to understand the challenges faced by communitybased DNSs in delivering a standardised service.

Methods We purposively sampled DNSs from communitybased respondents to a national survey $(n=25)$ according to four administrative regions of the national health service. We conducted focus groups and interviews using a semi-structured topic guide. Interviews were digitally recorded and transcribed into NVivo V.11 software for coding and analysis. Data analysis is on-going using thematic analysis.

Results Sixteen DNSs participated in 2 focus groups, and 8 interviews. Preliminary analysis suggested elements of the role presented a challenge. As DNSs require their Collaborative Practice Agreement to be signed off by each GP using their service, they were currently unable to prescribe in the community. Despite describing this as 'frustrating', DNSs suggested that prescribing could remove opportunities for relationshipbuilding with GPs through discussion of medications. In the community, DNSs lacked the safety net of the hospital team to check things with, and had to work more autonomously, described as 'daunting'. Role understanding by other staff was another challenge; DNSs felt managers did not understand how the community role should work, which created difficulty when negotiating aspects of the role, including flexible working hours. The lack of a shared record between settings meant patient information from hospital appointments was not readily accessible by DNSs at GP practices and vice versa. This made patient follow-up and case discussion difficult when DNSs were off-site. The absence of administrative support in the role, considered 'crucial', was also highlighted. Further interviews with community DNSs are ongoing.
Conclusion Community-based DNSs faced challenges presented by aspects of their role, their relationship with other staff, their work environment, and the available organisational infrastructure and resources. Although recent policy reforms in Ireland have focused on improving the integrated management of diabetes in the community, findings from this study suggest DNSs may need to be better supported to ensure delivery of a standardised model of diabetes care.

\section{Ethnicity 1}

\section{OP17 ETHNIC DIFFERENCES IN ILL HEALTH AND IN SOCIOECONOMIC INEQUALITIES IN HEALTH: POPULATION STUDY USING 2011 SCOTTISH CENSUS}

${ }^{1} \mathrm{M}$ Allik*, ${ }^{2} \mathrm{D}$ Brown, ${ }^{2} \mathrm{R}$ Dundas, ${ }^{2} \mathrm{AH}$ Leyland. 'Urban Big Data Centre, University of Glasgow, Glasgow, UK; ${ }^{2}$ MRC/CSO SPHSU, University of Glasgow, Glasgow, UK

\subsection{6/jech-2017-SSMAbstracts. 17}

Background Much has been written about high rates of poor health and health inequalities in Scotland, increasingly it is shown how these outcomes vary by ethnicity. Scottish Government has made a policy commitment to understand and address inequalities in health among minority ethnic groups. This study contributes to this by comparing health outcomes and socioeconomic inequalities in health across ethnicities

Methods Two self-reported health measures, poor general health and limiting long-term illness (LLTI), by 5 year age groups, ethnicity and area (Datazones; population mean $=815$, $\mathrm{sd}=275$ ) from the 2011 Scottish Census were examined. Ethnicity was self-reported and grouped into 9 main categories. This paper focused on the 5 largest groups: White Scottish $(\mathrm{n}=4,445,678)$, White British $(\mathrm{n}=417,109)$, White Irish $(\mathrm{n}=54,090)$, Other White $(\mathrm{n}=167,530)$ and Asian $(n=140,678)$ and ages $0-64$. Deprivation was measured using Census based indices and SIMD. Age standardised rates of ill health per 1000 people were calculated for these groups and by deprivation quintiles. Inequalities by area deprivation were measured using the slope index of inequality (SII).

Results For ages 0-64 the standardised rates are lowest for Other Whites and highest for White Scottish for both measures of ill health (LLTI rates respectively 89.1 and 134.9). Differences are greatest for younger adults, LLTI rate for Other Whites aged $15-29$ is 32.4 , but for White Scottish 71.7 , for ages 30-44 these rates are 63.6 and 124.2 respectively. On average White Scottish had poorer health than White British and Irish, both of who are also least likely to live in the most deprived areas. For ages 0-64 inequalities in health were highest for White Scottish (for LLTI the $\mathrm{SII}=164.4$, 95\% CI=163.1-165.7), but not much lower for White British $(\mathrm{SII}=150.8, \quad \mathrm{CI}=146.2-155.4)$ and Irish ( $\mathrm{SII}=145.2, \mathrm{CI}=133.6-156.8)$. Inequalities were much lower among Asians $(\mathrm{SII}=74.2, \mathrm{CI}=64.1-84.1)$ and among Other Whites $(\mathrm{SII}=59.3, \quad \mathrm{CI}=51.3-66.9)$. Differences in health inequalities between ethnicities were greatest for ages 30-44. Ill health and inequalities among Asians increased more rapidly for older ages and were similar to White Scottish for those $60+$.

Results White Scottish have poorer health compared to other ethnicities, but are also more likely to live in deprived areas compared to White British and Irish. Deprivation affects the health of all ethnicities, but much less so for some groups. 
Health inequalities are highest among White Scottish, similar for British and Irish, and significantly lower for Asians and Other Whites. This suggests that cultural and/or behavioural factors may reduce the effect of material deprivation on health.

\section{OP18 ETHNIC VARIATIONS IN INFANT MORTALITY IN ENGLAND AND WALES 2006-2012: A NATIONAL COHORT STUDY USING ROUTINELY COLLECTED DATA}

Y Li*, M Quigley, J Hollowell. Policy Research Unit in Maternal Health and Care, National Perinatal Epidemiology Unit, Nuffield Department of Population Health, University of Oxford, Oxford, UK

\subsection{6/jech-2017-SSMAbstracts. 18}

Background Marked ethnic variations in infant mortality have been documented in England and Wales. The reasons why certain ethnic groups have higher rates remain generally unknown. We aimed to evaluate ethnic variations in infant mortality, adjusting for deprivation and mother's migration status and to explore the contribution of preterm birth to these variations.

Methods Routinely collected birth and death registration data on all singleton live births (gestational age $\geq 22 \mathrm{w}$ ) in England and Wales, 2006-2012 were linked to other routine data on infant's ethnicity and gestational age. We analysed infant mortality by ethnic group using logistic regression in STATA to adjust for maternal sociodemographic characteristics (age, area deprivation, migration status and marital status/registration type) and gestational age.

Results In the 4,634,932 births analysed, crude infant mortality rates were highest in Pakistani, Black Caribbean, Black African, and Bangladeshi infants (6.92, 6.00, 5.17 and 4.40 per 1000 live births, respectively vs. 2.87 in White British infants). Adjustment for maternal sociodemographic characteristics changed the results little. Further adjustment for gestation significantly attenuated the risk in Black African (OR 1.17, 95\% CI 1.06-1.29) and Caribbean (OR 1.02, 95\% CI 0.89-1.17) but not in Pakistani (OR 2.32, 95\% CI 2.15-2.50), Bangladeshi (OR 1.47, 95\% CI 1.28-1.69), and Indian infants (OR 1.24, 95\% CI 1.11-1.38).

The association between ethnicity and infant mortality differed significantly between term and preterm infants $(p<0.001$ for interaction). In term infants, all three South Asian groups had a higher risk not explained by maternal characteristics or gestation. In preterm infants, adjustment for gestational age $(<28,28-31,32-33,34-36$ weeks) fully explained the higher risks in Black African and Caribbean infants, but not in Pakistani and Bangladeshi infants. A sensitivity analysis excluding deaths due to congenital anomalies did not fully explain the excess risk in Pakistani infants.

Conclusion South Asian and Black infants have higher infant mortality compared with White British infants which is not explained by deprivation, migration status or other maternal characteristics. A higher proportion of infants born at younger gestational age appears to explain the increased risks in preterm Black African and Caribbean infants but not in the South Asian groups.

This large national study provides insight into ethnic inequalities in early life to inform policy, practice, and future research. Findings suggest that strategies targeting the prevention and management of preterm birth in Black African and Caribbean mothers and babies would help to reduce ethnic inequalities in infant mortality. Different strategies may be required in South Asian groups.

\section{OP19 ROMA POPULATIONS AND HEALTH INEQUALITIES: A SYSTEMATIC SCOPING REVIEW OF MULTIPLE INTERSECTING DETERMINANTS}

LC Orton* ${ }^{*}$ R Anderson de Cuevas. Public Health and Policy, University of Liverpool, Liverpool, UK

\subsection{6/jech-2017-SSMAbstracts. 19}

Background There is growing evidence that Roma populations in Europe experience significantly poorer health-including higher rates of communicable and non-communicable diseases, poorer self-rated, child and maternal health-and shorter life expectancy than majority populations. Health outcomes are often worse than for others in similar social positions, suggesting the differences are not simply the effect of poverty. Roma women are thought to be worst affected. However, data pertaining to the multiple social inequalities affecting Roma populations, their interaction and cumulative effect on health over time, have yet to be explored. The aim of this paper is to critically review the state and shape of the research evidence on the multiple intersecting determinants of health for Roma populations in Europe.

Methods Studies were identified by searching four bibliographic databases (MEDLINE, Science Citation Index, Social Sciences Citation Index and Scopus), screening reference lists, consulting key informants and searching organisational websites. Any empirical studies (quantitative and qualitative) that explored more than one influencing factor in the pathway to poor relative health for Roma populations residing in the 53 countries of the WHO European region were included. Two reviewers carried out screening, data extraction and quality appraisal; disagreements were discussed by the wider team. This was followed by a narrative synthesis to explore the interactive effects of multiple axes of inequality.

Results From 2043 bibliographic records, 37 studies met our inclusion criteria. The median number of influencing factors explored through qualitative and quantitative methodologies was 2 (range 1-5) and 3 (range 1-11) respectively. Quantitative studies focus most strongly on factors that are welldefined and relatively easily turned into health indicators using available datasets, namely: poverty (10 studies) and other indicators of socio-economic status including education (13 studies), housing (9 studies) and employment (9 studies). Very few studies unpick interactive effects; instead testing each factor's contribution to poor health independently. On the other hand, qualitative studies tended to explore "Roma culture" (11 studies) and the impact of discrimination/racism on health behaviour (10 studies). There is a lack of gender-disaggregated data and little analysis of the impact of social policy on the health of Roma populations.

Conclusion Evidence for associations between determinants and health outcomes among European Roma populations is patchy.Research needs to pay more attention to the health impact of social policies that have the potential to reinforce (as well as mitigate) the exclusion of Roma populations and the disadvantages experienced by women. 